\title{
Intraoperative Disseminated Intravascular Coagulation During Thoracolumbar Decompression in a Patient With Metastatic Carcinoma of the Prostate: Etiology, Diagnosis, and Treatment
}

\author{
Mahmood Rafiq ${ }^{\text {a, d }}$, Joseph D. Tobias ${ }^{\text {a, b, c }}$
}

\begin{abstract}
Various factors may be responsible for disturbances of coagulation function during the perioperative period. Disseminated intravascular coagulation or coagulopathy (DIC) results from the widespread activation of the clotting cascade on the endothelial surface throughout the microvasculature that results in the formation of thrombin and fibrin. Although an uncommon cause of intraoperative bleeding, previous reports have noted the occurrence of DIC in association with adenocarcinoma of the prostate. We present a 62-year-old man with known metastatic carcinoma of prostate, who presented with rapidly progressing paraplegia and incontinence. During an emergent posterior thoracolumbar decompression and instrumentation, he developed excessive blood loss and DIC. The potential etiologies for intraoperative DIC are reviewed, the diagnosis is discussed, and perioperative management strategies are presented.
\end{abstract}

Keywords: Disseminated intravascular coagulation; Bleeding; Adenocarcinoma; Prostate

\section{Introduction}

Carcinoma of the prostate is the most common cancer and the third most frequent cause of death from cancer in the males, following only cancer of the lung and colorectal cancer [1]. While metastatic disease and local tumor invasion represent the most common sequelae of the disease process, disruptions of coagulation with disseminated intravascular coagulation

Manuscript accepted for publication August 13, 2015

aDepartment of Anesthesiology \& Pain Medicine, Nationwide Children's Hospital, Columbus, OH, USA

bepartment of Anesthesiology \& Pain Medicine, The Ohio State University College of Medicine, Columbus, OH, USA

'Department of Pediatrics, The Ohio State University College of Medicine, Columbus, OH, USA

${ }^{\mathrm{d} C}$ Corresponding Author: Mahmood Rafiq, Department of Anesthesiology \& Pain Medicine, Nationwide Children's Hospital, 700 Children's Drive, Columbus, OH 43205, USA. Email: Mahmood.Rafiq@Nationwidechildrens.org

doi: http://dx.doi.org/10.14740/jmc2264w
(DIC) have also been reported. In addition to gastric or pancreatic cancers, prostate adenocarcinoma is one of the most common solid malignancies responsible for inducing DIC [2, 3]. The incidence of DIC in association with prostate cancer has been reported to be as high as 10-30\% [2-5].

Up to $80-100 \%$ of patients with advanced prostate cancer develop metastatic disease involving the bones with the spine being the most common site $[6,7]$. Compression of the spinal cord can result from either vertebral collapse resulting from tumor invasion into the vertebral body, or by extradural tumor growth anywhere along the spinal cord [8]. Motor weakness in the lower extremities may arise and is often acute and can progress rapidly to paraplegia. In such cases, surgical intervention provides the greatest chance for the limitation of neurologic deficit. Posterior laminectomy remains the standard surgical approach for most decompressions. The manipulation of the tumor during the surgery may facilitate the passage of procoagulant substances into the blood stream [9]. We present a 62-year-old man with known metastatic carcinoma of prostate, who presented with rapidly progressing paraplegia and incontinence. During an emergent posterior thoracolumbar decompression and instrumentation, he developed excessive blood loss and DIC intraoperatively. The potential etiologies for intraoperative DIC are reviewed, the diagnosis is discussed, and perioperative management strategies are presented.

\section{Case Report}

The patient was cared for at King Fahad Medical Center (Riyadh, Saudi Arabia). Institutional Review Board approval is not required by the institution for publication of case reports. A 68-year-old, $64.2 \mathrm{~kg}$ man with chronic hypertension was admitted with urinary retention for which transurethral resection of the prostate (TURP) was performed uneventfully under spinal anesthesia. On the fifth postoperative day, the patient developed motor weakness in both lower limbs and urinary incontinence. An urgent MRI/CT showed compression fractures of $\mathrm{T}_{6}$ and $\mathrm{L}_{1}$ and retropulsion of the posterior cortex into the spinal canal with cord compression (Fig. 1). Diffuse osseous metastatic disease was noted throughout the axial skeleton. An urgent posterior decompression and instrumentation of thoracolumbar spine was planned. Medications included androgen deprivation therapy (leuprolide), amlodipine $10 \mathrm{mg}$ once a 

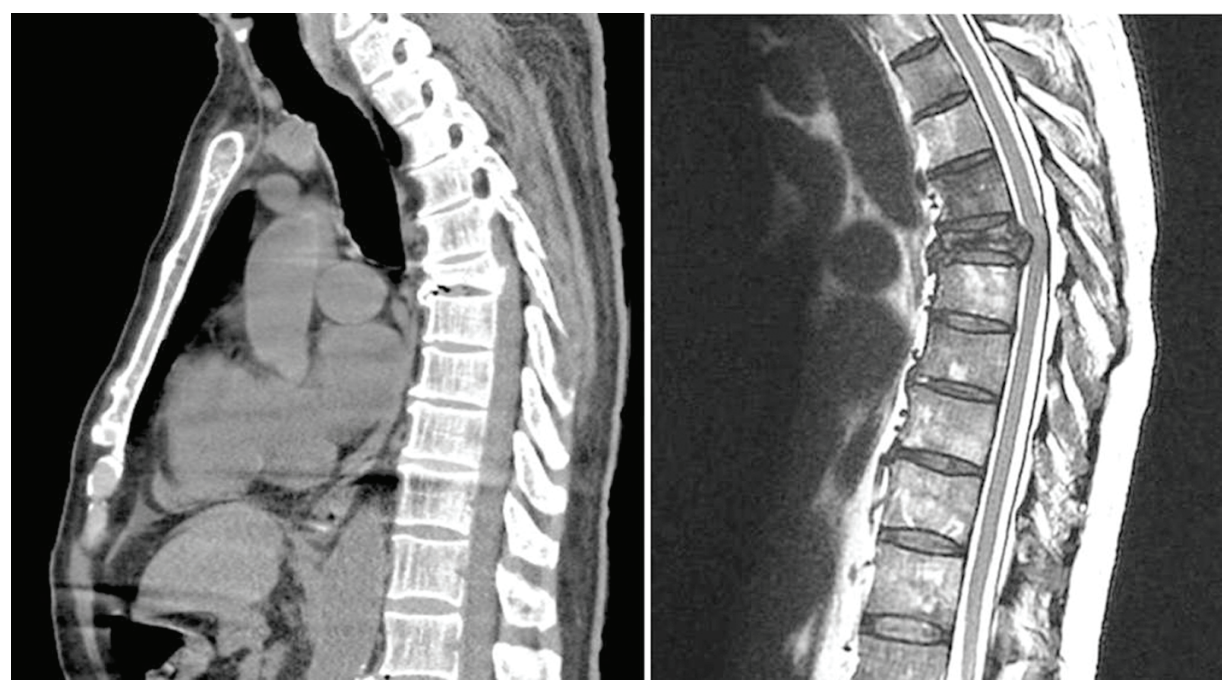

Figure 1. Computed tomography imaging (left) and magnetic resonance imaging (right) showing compression fractures of $T_{6}$ and retropulsion of the posterior cortex into the spinal canal with cord compression.

day and atenolol $50 \mathrm{mg}$ once a day. Dexamethasone $(16 \mathrm{mg}$ every $6 \mathrm{~h}$ ) was initiated with the change in neurologic function. He denied other respiratory or cardiac diseases. Preoperative electrocardiogram and laboratory analysis were within normal range. The chest radiograph was found to be normal apart from the $\mathrm{T}_{6}$ compression fracture. The coagulation studies revealed a prothrombin time (PT) of $13 \mathrm{~s}$, international normalized ratio (INR) of 1.1, and partial thromboplastin time (PTT) of $35 \mathrm{~s}$. The hemoglobin was $11.5 \mathrm{~g} / \mathrm{dL}$ with a platelet count of 178,000 $\mathrm{mm}^{3}$. The patient was transported to the operating room and standard monitoring was applied. Anesthesia was induced with midazolam (1 mg), propofol $(120 \mathrm{mg})$, and fentanyl $(150 \mu \mathrm{g})$. Neuromuscular blockade was provided by rocuronium $(50$ $\mathrm{mg}$ ) followed by endotracheal intubation. Two 16-gauge intravenous cannulas and a 20 -gauge radial arterial cannula were established. The patient was positioned prone and all pressure points were padded. Anesthesia was maintained with propofol and remifentanil infusion with the bispectral index values ranging from 40 to 50. Tranexamic acid was administered as a bolus of $10 \mathrm{mg} / \mathrm{kg}$ followed by an infusion of $1 \mathrm{mg} / \mathrm{kg} / \mathrm{h}$. Surgery proceeded uneventfully until the completion of the thoracolumbar decompression with blood loss of approximately 500 $\mathrm{mL}$. The hemoglobin was $10.4 \mathrm{~g} / \mathrm{dL}$. Thoracic instrumentation from $\mathrm{T}_{4-8}$ was started $2 \mathrm{~h}$ from the beginning of anesthesia. At this time, rapid blood loss (about 2,000 mL) and absence of clot formation at the surgical site was noted. A complete blood count and coagulation profile revealed hemoglobin $6.8 \mathrm{~g} / \mathrm{dL}$, platelet count $49,000 \mathrm{~mm}^{3}$, PT $28 \mathrm{~s}$, INR 2.2, aPTT $50.4 \mathrm{~s}$, and fibrinogen $1.2 \mathrm{~g} / \mathrm{L}$. The D-dimer was elevated at $15.4 \mu \mathrm{g} / \mathrm{mL}$. A diagnosis of DIC was made and management included the administration of blood and blood products including packed red blood cells, fresh frozen plasma, cryoprecipitate, and platelet concentrates. Albumin 5\% was used to maintain intravascular volume. No vasopressor or inotrope therapy was required during the procedure. Clinically, the coagulopathy improved after the blood and blood products were administered. Repeat hemoglobin was $9.8 \mathrm{~g} / \mathrm{dL}$ with a platelet count of $75,000 / \mathrm{mm}^{3}$.
The surgical procedure lasted $4 \mathrm{~h} 40 \mathrm{~min}$ with completion of $\mathrm{T}_{4-8}$ and $\mathrm{T}_{11}-\mathrm{L}_{3}$ instrumentation. Morphine was administered for postoperative analgesia, the patient was turned prone and his trachea was extubated when he was awake. During the procedure, the patient received 6 units of packed RBCs, 12 units of pooled platelets, 12 units of fresh frozen plasma, 10 units of cryoprecipitate in addition to $1 \mathrm{~L}$ of $5 \%$ albumin and $2 \mathrm{~L}$ of crystalloid. He was transferred to a high dependency unit for further management where he had further decreases in hemoglobin and platelet count which required the transfusion of additional units of red blood cells, fresh frozen plasma, platelets, and cryoprecipitate. The patient's hemoglobin, coagulation function, and platelet count stabilized $6 \mathrm{~h}$ after completion of the surgery. He was transferred to the inpatient ward $24 \mathrm{~h}$ later in stable condition with minimal surgical site drain collection. He did not require any further transfusion of blood and blood products and 5 days post-surgery, he had normal bowel, bladder, and motor function as well as a normal coagulation profile.

\section{Discussion}

Various factors may be responsible for disturbances of coagulation function during the perioperative period, most commonly large volume blood loss, necessitating resuscitation with the subsequent dilution of coagulation factors and platelets [10]. In the absence of exsanguination, other etiologies must be considered when excessive bleeding with inadequate clot formation is noted. In our patient, excessive bleeding started before the administration of allogeneic blood products suggesting that other etiologic factors were present.

DIC results from the widespread activation of the clotting cascade on the endothelial surface throughout the microvasculature that results in the formation of thrombin and fibrin [11]. The damage to the microcirculation compromises tissue blood flow and can ultimately lead to multiple system organ failure. Activation of the coagulation process consumes clot- 
ting factors and platelets, resulting in the clinical picture of coagulopathy with excessive bleeding. DIC does not occur as a primary disease process, but only as a complicating factor from or response to another underlying condition. The clinical manifestations can be overt and severe in some cases or milder and more insidious in others. The diagnosis of DIC depends on the findings of characteristic laboratory tests and clinical background (see below). Management includes treatment of the primary disease process (sepsis, shock), reversal of associated end-organ dysfunction (hypotension, hypoxemia, acidosis), and correction of the coagulation disturbances with the administration of blood and blood products.

While disturbances of coagulation are frequent in oncology patients, the presence of overt DIC is uncommon and its intraoperative presentation is even rarer. However, the association of DIC with advanced adenocarcinoma of the prostate has been previously reported in the literature, at times being the initial presentation of the malignancy $[4,5]$. In these circumstances, DIC is postulated to result from the release of procoagulant substances, such as tissue factor, into the blood stream [9]. The manipulation of the tumor during the surgery likely accelerates the passage of these procoagulant substances to the blood stream. As was the case with our case, patients with spinal metastasis from prostate cancer exhibiting rapidly progressive neurological deterioration or demonstrating evidence of spinal mechanical instability require urgent surgical intervention. Because of the propensity of prostate cancer to metastasize to bone, most of the patients will have evidence of multiple spinal lesions thereby predisposing them to the release of procoagulant substances during surgery with the development of DIC. As such, anesthesia providers should be aware of the potential for this complication to occur intraoperatively and maintain a high index of suspicion to allow for early diagnosis and treatment.

The diagnosis of DIC includes both clinical and laboratory information. Most importantly, unexplained bleeding which cannot be attributed to a surgical cause with the absence of clot formation should prompt a laboratory investigation to diagnosis DIC. There is no single laboratory test that can establish or rule out the diagnosis of DIC. However, a combination of tests when repeated in a patient with a clinical condition known to be associated with DIC can be used to diagnose the disorder with reasonable certainty [5, 11-14]. Screening assays for hemostatic function including the PT, PTT, INR and platelet count provide important evidence of the degree of coagulation factor consumption and activation. An analysis of reports of patients with DIC suggests that the laboratory abnormalities in decreasing order of frequency included thrombocytopenia, elevated fibrin degradation products, prolonged PT, prolonged aPTT, and a low fibrinogen concentration. A reduction in the platelet count or a clear downward trend over subsequent measurements is a sensitive, though not specific, sign of DIC. In our case there was progressive fall in the platelet count during the operative procedure. Thrombocytopenia is noted in up to $98 \%$ of DIC cases with the platelet count being less than $50,000 / \mathrm{mm}^{3}$ in approximately $50 \%$ of cases [15]. A stable platelet count suggests that thrombin formation has stopped.

Fibrin degradation products including D-dimers should not be considered as diagnostic of DIC, but as a useful indica- tor of the DIC process with active lysis of clot that has been formed in the microcirculation and the release of the degradation products from fibrin. The presence of fibrin degradation products in the presence of a decreasing platelet count is highly suggestive of DIC. The PT or aPTT is prolonged in approximately $50-60 \%$ of cases. Measurement of fibrinogen has been widely advocated as a useful tool for the diagnosis of DIC, but a low fibrinogen concentration is least sensitive of the routine coagulation values. Although not routinely obtained, the serum concentrations of the natural anticoagulants, anti-thrombin III and protein $\mathrm{C}$ are often reduced in $\mathrm{DIC}$, and may have some prognostic significance. These values have been included by the ISTH Sub-Committee of the Scientific and Standardization Committee (SSC) on DIC into a scoring system [16, 17].

The cornerstone of the treatment of DIC remains treatment and reversal of the underlying condition. Correction of secondary metabolic consequences of the primary disease process (acidosis and hypotension) is also indicated as well as reversal of associated conditions which may aggravate coagulation disturbances (hypothermia and hypocalcemia). Bleeding and alterations in coagulation function require the administration of blood and blood products based on the coagulation profile with the administration of platelets to treat thrombocytopenia (generally for a platelet count less than $50,000 / \mathrm{mm}^{3}$ ), fresh frozen plasma to treat elevated PT, INR, and PTT or the administration of cryoprecipitate or purified fibrinogen concentrations to treat a low fibrinogen level (less than $100 \mathrm{mg} / \mathrm{dL}$ ). It may be necessary to use large volumes of fresh frozen plasma to correct the coagulation defect. While initial doses of $15 \mathrm{~mL} / \mathrm{kg}$ of fresh frozen are generally suggested, there is evidence that a dose of $30 \mathrm{~mL} / \mathrm{kg}$ produces more complete correction of coagulation factor levels [18]. Adjunctive therapy with tranexamic acid may be added to control excessive thrombolysis which may exacerbate the bleeding of DIC $[19,20]$. In extreme cases, when conventional therapy fails, anecdotal success has been reported with recombinant factor VIIa in patients with DIC and life-threatening bleeding $[21,22]$. However, the safety of this agent in the setting of DIC has not been studies [23]. The administration of packed red blood cells should be guided according to the estimated blood loss and the values obtained from serial hematocrits. Given these concerns, there has been ongoing interest in preoperative therapies including vertebral tumor embolization to decrease perioperative blood loss, which can be considered in select cases [24]. Other selective therapies that have been anecdotally reported in the literature to treat DIC associated with prostatic cancer include abiraterone acetate, ketoconazole, estrogen analogues, docetaxel, cisplatin, mitoxantrone, and samarium [25-27].

In summary, we present the unusual intraoperative occurrence of DIC related to adenocarcinoma of the prostate. When unexplained bleeding without clot formation occurs intraoperatively, DIC should be considered high on the differential diagnosis in patients with prostatic cancer. Prompt diagnosis, an understanding of the underlying mechanisms of disease processes, and institution of appropriate therapy are essential for a favorable outcome. While the primary therapy is reversal of the underlying disease process, secondary treatments include reversal of associated end-organ dysfunction (hypotension, hypoxemia, and acidosis), and correction of the coagulation 
disturbances with the administration of blood and blood products.

\section{References}

1. Attard G, Parker C, Eeles RA, Schroder F, Tomlins SA, Tannock I, Drake CG, et al. Prostate cancer. Lancet. 2015.

2. Adamson AS, Francis JL, Witherow RO, Snell ME. Coagulopathy in the prostate cancer patient: prevalence and clinical relevance. Ann R Coll Surg Engl. 1993;75(2):100104.

3. McKechnie J. Prostatic carcinoma presenting as a haemorrhagic diathesis after dental extraction. Br Dent J. 1989;166(8):295-296.

4. Tagnon HJ, Whitmore WF, Jr., Shulman NR. Fibrinolysis in metastatic cancer of the prostate. Cancer. 1952;5(1):912.

5. Spector JI, Zimbler H. Carcinoma of the prostate presenting as acute disseminated intravascular coagulation. CMAJ. 1987;136(6):570.

6. Bubendorf L, Schopfer A, Wagner U, Sauter G, Moch H, Willi N, Gasser TC, et al. Metastatic patterns of prostate cancer: an autopsy study of 1,589 patients. Hum Pathol. 2000;31(5):578-583.

7. Mehra R, Kumar-Sinha C, Shankar S, Lonigro RJ, Jing X, Philips NE, Siddiqui J, et al. Characterization of bone metastases from rapid autopsies of prostate cancer patients. Clin Cancer Res. 2011;17(12):3924-3932.

8. Rubin H, Lome LG, Presman D. Neurological manifestation of metastatic prostatic carcinoma. J Urol. 1974;111(6):799-802.

9. Kampel LJ. Challenging problems in advanced malignancy: Case 2. Disseminated intravascular coagulation in metastatic hormone-refractory prostate cancer. J Clin Oncol. 2003;21(16):3170-3171.

10. Grottke O, Fries D, Nascimento B. Perioperatively acquired disorders of coagulation. Curr Opin Anaesthesiol. 2015;28(2):113-122.

11. Levi M. Disseminated intravascular coagulation. Crit Care Med. 2007;35(9):2191-2195.

12. Bick RL. Disseminated intravascular coagulation: objective clinical and laboratory diagnosis, treatment, and assessment of therapeutic response. Semin Thromb Hemost. 1996;22(1):69-88.

13. Levi M, Ten Cate H. Disseminated intravascular coagulation. N Engl J Med. 1999;341(8):586-592.

14. Toh CH, Dennis M. Disseminated intravascular coagulation: old disease, new hope. BMJ. 2003;327(7421):974977.

15. Spero JA, Lewis JH, Hasiba U. Disseminated intravascular coagulation. Findings in 346 patients. Thromb Hae- most. 1980;43(1):28-33.

16. Taylor FB, Jr., Toh CH, Hoots WK, Wada H, Levi M. Towards definition, clinical and laboratory criteria, and a scoring system for disseminated intravascular coagulation. Thromb Haemost. 2001;86(5):1327-1330.

17. Toh CH, Hoots WK. The scoring system of the Scientific and Standardisation Committee on Disseminated Intravascular Coagulation of the International Society on Thrombosis and Haemostasis: a 5-year overview. J Thromb Haemost. 2007;5(3):604-606.

18. Levi M, Toh CH, Thachil J, Watson HG. Guidelines for the diagnosis and management of disseminated intravascular coagulation. British Committee for Standards in Haematology. Br J Haematol. 2009;145(1):24-33.

19. Cooper DL, Sandler AB, Wilson LD, Duffy TP. Disseminated intravascular coagulation and excessive fibrinolysis in a patient with metastatic prostate cancer. Response to epsilon-aminocaproic acid. Cancer. 1992;70(3):656-658.

20. Tsuji T, Morikawa K, Hirano T, Yamasaki H, Tsuda H. [Effective treatment with tranexamic acid for chronic disseminated intravascular coagulation associated with aortic dissection]. Rinsho Ketsueki. 2013;54(8):769-771.

21. Nohira T, Osakabe Y, Suda S, Takahashi C, Tanaka A, Ikeda K, Ikeda T, et al. Successful management by recombinant activated factor VII in a case of disseminated intravascular coagulopathy caused by obstetric hemorrhage. J Obstet Gynaecol Res. 2008;34(4 Pt 2):623-630.

22. Prosper SC, Goudge CS, Lupo VR. Recombinant factor VIIa to successfully manage disseminated intravascular coagulation from amniotic fluid embolism. Obstet Gynecol. 2007;109(2 Pt2):524-525.

23. Goodnough LT, Shander AS. Recombinant factor VIIa: safety and efficacy. Curr Opin Hematol. 2007;14(5):504509.

24. Prabhu VC, Bilsky MH, Jambhekar K, Panageas KS, Boland PJ, Lis E, Heier L, et al. Results of preoperative embolization for metastatic spinal neoplasms. J Neurosurg. 2003;98(2 Suppl):156-164.

25. Gauthier H, Serrate C, Pouessel D, leMaignan C, Teixeira L, Culine S. Very fast recovery of acute disseminated intravascular coagulation with abiraterone acetate in a patient with bone metastases from castrate-resistant prostate cancer. Case Rep Oncol. 2014;7(3):625-627.

26. Fleyfel M, Leroy B, Plouvier F, Balin C, Marecaux O, Scherpereel P. [Favorable outcome of neoplastic disseminated intravascular coagulation treated with ketoconazole and estrogen derivative]. Ann Fr Anesth Reanim. 1991;10(4):406-408.

27. Avances C, Jacot W, Senesse P, Culine S. Prompt resolution of acute disseminated intravascular coagulation with docetaxel and cisplatin in hormone refractory prostate cancer. J Urol. 2002;168(4 Pt 1):1496. 